\title{
Diferencias de la población general en relación a los conoci- mientos, actitudes y conductas hacia el estigma en salud mental
}

\author{
Ana María Moreno Herrero*, Francisca Sánchez Sánchez y Manuel López de Lemus \\ Sarmiento \\ Complejo Hospitalario de Torrecárdenas, Almería, España
}

(Recibido 6 Octubre 2013; Aceptado 22 febrero 2014)

RESUMEN: Se estudia una muestra de 152 sujetos de la población de Almería capital con respecto al Estigma en Salud Mental con el objetivo de diseñar intervenciones anti-estigma específicas. Para ello se operativiza el Estigma en tres constructos: Conocimiento, Actitudes y Conducta utilizando las escalas MAKS, CAMI y RIBS respectivamente. Los resultados indican que los más jóvenes, menores de treinta y cinco años, son los que de forma significativa $(p<.05)$ cuentan con menos conocimientos en Salud Mental relacionado con el Estigma. Así mismo se encuentran diferencias significativas entre sexos en referencia a la intención de conductas estigmatizantes $(\mathrm{p}=.049)$ y de actitudes estigmatizantes $(\mathrm{p}=.006)$ siendo los hombres los que tienden a exhibir intención de conducta y actitudes más estigmatizantes. Estos resultados junto con otros que nos muestran una correlación significativa entre Conocimientos y Conducta y por otro lado entre Actitudes y Conducta, nos sirven de guía en el diseño de intervenciones encaminadas a reducir el estigma en salud mental.

Palabras clave: estigma, salud mental, población general.

\section{Differences in general population on knowledge, attitudes and behaviour related to mental health stigma}

\begin{abstract}
Stigma in mental health is studied in a sample of 152 subjects of Almería city population with the aim of designing specific anti-stigma campaigns. For this, Stigma is operatized in three constructs: Knowledge, Attitudes and Behaviour, using MAKS, CAMI and RIBS scales for these. Results show that the younger group, under thirty-five years old are less knowledgeable about mental health related to stigma, showing a significant difference $(\mathrm{p}<.05)$. Moreover, we found significant differences in gender regarding the intention of stigmatizing behaviour $(\mathrm{p}=.049)$ as well as attitudes $(\mathrm{p}=.006)$ where men have more stigmatizing behaviour and attitudes than women. These results together with those that show a significant correlation between knowledge and behaviour, as well as between attitudes and behaviour will be used as a guide to design interventions aimed to reduce stigma in mental health.
\end{abstract}

Keywords: stigma, mental health, general population. 


\section{INTRODUCCIÓN}

El estigma es un fenómeno conocido y presente en muchos ámbitos, y muy especialmente en el de la salud mental, campo en el que ha sido, y sigue siendo, una importante barrera; es una barrera relevante para la atención y la integración social de las personas afectadas por enfermedades mentales (López, 2012).

Las personas con enfermedad mental a menudo tienen que enfrentarse a un doble problema, por un lado a la sintomatología de la propia enfermedad, y por otro al estigma. Los problemas de salud mental todavía viven y se viven en el silencio, a menudo provocado por el miedo, la vergüenza o la incomprensión, en resumen, por el estigma social que los rodea y los ha acompañado durante siglos. Ese silencio impide que muchas personas afectadas busquen ayuda profesional y se recuperen.

Mientras el conocimiento científico avanza, la sociedad muy a menudo sigue anclada en estereotipos, que producen discriminación hacia las personas que padecen problemas de salud mental. Las actitudes de marginación y desprecio mantenidas a lo largo de la historia todavía perviven. Un error muy común es pensar que la enfermedad mental no es "algo que alguien tiene", sino "algo que alguien es". Se identifica completamente a la persona con la patología y se lanzan sobre ella todos los prejuicios generados por falsos mitos. Este estigma social es una carga añadida en la persona.

Las actitudes estigmatizantes hacia las personas con enfermedad mental tienen también un reflejo en la sociedad y en la convivencia diaria, y también en los servicios sanitarios y en las propias consultas de atención primaria y especializada (Magliano, Read, Patalano, Sagliocchi, Oliviero, D’Ambrosio y Cerrato, 2012). El estigma puede deteriorar el estado de salud de las personas y afectar su recuperación e incluso impedir la detección del problema.

Lo anteriormente descrito hace necesario operativizar de la forma más objetiva posible este fenómeno. El estigma, un concepto ampliamente utilizado, se compone de elementos cognitivos, emocionales y conductuales. Los elementos cognitivos se corresponden a la información y conocimiento que tenemos sobre los problemas de Salud Mental, los trastornos y enfermedades mentales, su incidencia y prevalencia, tratamiento y la forma de acceso a la asistencia sanitaria entre otros. Las actitudes se reflejan en elementos emocionales que suscitan ciertos grupos sociales basados en estereotipos normalmente negativas dando lugar a los prejuicios. Un conocimiento sesgado basado en estereotipos negativos se relaciona con actitudes emocionales así mismo negativas o prejuicios lo cual está interrelacionado con una conducta de discriminación hacia este colectivo (López, Laviana, Fernández, López, Rodríguez, y Aparicio, 2008). Nos encontramos ante un fenómeno social multidimensional con elementos cognitivos, emocionales y conductuales los cuales están interrelacionados entre sí. Algunos estudios señalan que la información se acepta en la medida que es congruente con las emociones o actitudes que se tiene hacia cierto colectivo social, determinando las conductas de discriminación. Sin embargo, las relaciones de causalidad son 
circulares y no siempre siguen una línea de actuación similar (Fazio y Olson, 2003). Así, el conocimiento que existe acerca de los mecanismos que subyacen a esta interrelación es limitado debido a su complejidad.

El Estigma ha sido conceptualizado en previos estudios como formado por tres constructos: Conocimiento (Ignorancia), Actitudes (Prejuicio) y Conducta (Discriminación) (Thorndicroft, 2004).

El conocimiento que la población general tiene del enfermo de Salud Mental es por un lado la transmitida por medios de comunicación los cuales también tienen un conocimiento sesgado sobre la materia y la de estereotipos negativos anclados en la sociedad, estando relacionada la falta de conocimiento en Salud Mental con más estigma personal y más temor e incomodidad en contacto con personas con un problema de Salud Mental (Brändli, 1999).

Existe evidencia que señala que el componente de Conocimiento "puede ser significativo por sí mismo como mediador en cambios actitudinales y de comportamiento en relación al estigma" (Wolff, Pathare y Craig, 1996).

Así mismo, una evaluación del Conocimiento previo y posterior a una intervención para disminuir el estigma puede ayudar a identificar ciertas lagunas de conocimiento y a evaluar la efectividad de la intervención en cambios de conocimiento, actitud y comportamiento en la población general con respecto al estigma.

El presente estudio, sigue una línea de investigación donde se trata de ampliar y comprender este fenómeno del estigma en Salud Mental en sus distintos componentes así como mejorar la compresión de la relación entre los constructos que lo forman. Para ellos se han elegido los cuestionarios MAKS, CAMI y RIBS que evalúan conocimientos, actitudes y conducta relacionadas con el estigma en Salud Mental para población general respectivamente.

Hasta ahora las medidas utilizadas para medir el estigma en Salud Mental en la población general han sido muy generales o medían el estigma desde la propia percepción del enfermo mental. Estas medidas no recogen la complejidad de un fenómeno como el Estigma. Para ello se han elegido para este estudio unos instrumentos de evaluación que miden Estigma en sus diferentes constructos (MAKS, CAMI y RIBS), siendo pioneros en el uso de MAKS y RIBS en español.

Pretendemos inicialmente ampliar nuestro conocimiento sobre el concepto Estigma, sus componentes y mecanismos asociados aún poco claros, y como éstos se manifiestan en la sociedad almeriense. Esto significa un primer paso en la aproximación al objetivo de diseñar intervenciones específicas a adecuadas a las necesidades de nuestra población y así posteriormente evaluar el impacto de la intervención y seguir recabando información sobre qué tipo de intervención es más efectiva. Así mismo, obtener información sobre los tipos de intervención en relación a la duración de la misma, métodos empleados y población diana.

Esta línea de investigación se orienta a reducir el estigma asociado a los problemas de Salud Mental y promover una mayor integración de los distintos colectivos para mejorar la calidad de vida de las personas con problemas de salud mental dentro de la sociedad. 


\section{OBJETIVOS}

Los objetivos que nos proponemos en este estudio son:

a. Medir los niveles de conocimientos sobre salud mental en relación al estigma en la población general y las diferencias entre grupos.

b. Medir los niveles de actitudes sobre salud mental en relación al estigma en la población general y las diferencias entre grupos.

c. Medir los niveles de conductas sobre salud mental en relación al estigma en la población general y las diferencias entre grupos.

d. Ampliar nuestros conocimientos y compresión sobre el fenómeno del Estigma en sus diferentes componentes y su interacción entre sí.

e. Obtener la información necesaria para diseñar futuras intervenciones antiestigma específicas.

\section{METODOLOGÍA}

La muestra estuvo compuesta por 152 participantes elegidos aleatoriamente de la población general almeriense, excluyendo sanitarios de profesión. La media de edad fue de 35,28 años $(D T=11,01)$, de los cuales 75 eran mujeres $(49,7 \%)$ y 77 hombres $(50,3 \%)$. En relación al estado civil: 88 estaban solteros y 56 casados (se recogieron algunos casos más, divorciados, separados y viudos, que se han descartado para esta variable por ser muy pocos casos). En cuanto al nivel académico: 20 sujetos tienen estudios básicos (13,1\%), 50 estudios medios $(32,7 \%)$ y 82 estudios superiores $(53,6 \%)$. Finalmente respecto a la situación laboral: 89 sujetos estaban activos $(58,2 \%)$ y 44 parados $(28,8 \%)$, el resto de sujetos no se han utilizados para este análisis por encontrar pocos casos (autónomo 5 , jubilado 1 , estudiante 1 y otros 2 ).

\section{INSTRUMENTOS}

Mental Health Knowledge Schedule (MAKS) (Evans-Lacko et al. 2010). La escala sobre conocimientos en Salud Mental ) incluye dominios de conocimiento con evidencia de relevancia en relación a la reducción del estigma, el cual puede ser utilizado junto con medidas de las actitudes y las conductas en la población general. Incluye seis áreas de conocimiento en salud mental en relación al estigma: búsqueda de ayuda, reconocimiento, apoyo, empleo, tratamiento y recuperación, y seis ítems que preguntan acerca de conocimiento sobre enfermedades mentales. Cuenta con una fiabilidad test-retest del 0,71, siendo pioneros en su utilización en español, así como de la escala RIBS (Escala de Conducta Informada e Intencionada)

Reported and intended Behaviour Scale (RIBS) (Evans-Lacko et. al, 2011). Evalúa conducta intencionada y consumada, basado en The Star Social Distance Scale (Escala Estrella de Distancia Social) la cual está diseñada para evaluar a la 
población general (Evans-Lacko et al., 2010). Puede ser usada en conjunto con medidas de conocimiento y actitudes sobre estigma en salud mental.

Community Attitudes toward the Mentally Ill (CAMI) (Taylor y Dear, 1981). Mide las actitudes de la comunidad hacia los enfermos mentales. Es utilizada ampliamente, junto con medidas de conocimiento y conducta, en el tema que nos ocupa en la población general.

\section{RESULTADOS}

Se analiza una muestra de 152 sujetos, comparando las diferencias de medias de los tres cuestionarios (Conocimiento, Actitudes y Conducta) entre los diferentes niveles de las variables Sexo, Edad, Estado Civil, Nivel Académico y Situación Laboral. Analizados mediante el paquete estadístico SPSS15 aplicando la T de Student para comparar medias y el ANOVA cuando existen más de dos niveles (tabla 1).

Tabla 1. Comparación de Medias en Relación a los Tres Cuestionarios (MAKS, RIBS y CAMI) en Función de las VariablesEstudiadas (T de Student y ANOVA).

\begin{tabular}{|c|c|c|c|c|c|c|c|c|c|}
\hline & & & $n$ & $M$ & D.T. & $t / F$ & $g l$ & $p$ & $\begin{array}{l}d / \\
\eta^{2}\end{array}$ \\
\hline \multirow{11}{*}{ MAKS } & \multirow[t]{2}{*}{ Edad } & $<=35$ & 76 & 20,83 & 3,02 & \multirow[t]{2}{*}{$-3,545$} & \multirow[t]{2}{*}{151} & \multirow[t]{2}{*}{.001} & \multirow[t]{2}{*}{-0.66} \\
\hline & & $>35$ & 77 & 22,78 & 3,74 & & & & \\
\hline & \multirow[t]{2}{*}{ Sexo } & $\mathrm{H}$ & 77 & 21,43 & 3,87 & \multirow[t]{2}{*}{$-1,478$} & \multirow[t]{2}{*}{144} & \multirow[t]{2}{*}{.142} & \multirow[t]{2}{*}{-0.33} \\
\hline & & $\mathrm{M}$ & 75 & 22,27 & 3,08 & & & & \\
\hline & \multirow[t]{2}{*}{ Estado Civil } & $\mathrm{S}$ & 88 & 21,09 & 3,42 & \multirow[t]{2}{*}{$-3,226$} & \multirow[t]{2}{*}{142} & \multirow[t]{2}{*}{.002} & \multirow[t]{2}{*}{-0.33} \\
\hline & & $\mathrm{C}$ & 56 & 22,96 & 3,36 & & & & \\
\hline & \multirow{3}{*}{$\begin{array}{c}\text { Nivel } \\
\text { Académico }\end{array}$} & Graduado & 20 & 22,10 & 3,99 & \multirow[t]{3}{*}{0,140} & \multirow[t]{3}{*}{2} & \multirow[t]{3}{*}{.869} & \multirow[t]{3}{*}{.002} \\
\hline & & Bachiller & 50 & 21,96 & 3,03 & & & & \\
\hline & & Universitario & 82 & 21,71 & 3,70 & & & & \\
\hline & \multirow{2}{*}{$\begin{array}{l}\text { Situación } \\
\text { Laboral }\end{array}$} & Activo & 89 & 22,22 & 3,63 & \multirow[t]{2}{*}{1,166} & \multirow[t]{2}{*}{131} & \multirow[t]{2}{*}{.246} & \multirow[t]{2}{*}{0.33} \\
\hline & & Parado & 44 & 21,48 & 3,14 & & & & \\
\hline \multirow{11}{*}{ RIBS } & \multirow[t]{2}{*}{ Edad } & $<=35$ & 76 & 14,12 & 4,02 & \multirow[t]{2}{*}{1,459} & \multirow[t]{2}{*}{151} & \multirow[t]{2}{*}{.147} & \multirow[t]{2}{*}{0.25} \\
\hline & & $>35$ & 77 & 13,17 & 4,03 & & & & \\
\hline & \multirow[t]{2}{*}{ Sexo } & $\mathrm{H}$ & 77 & 13,01 & 4,24 & $-1,99$ & 150 & .049 & $-0,28$ \\
\hline & & $\mathrm{M}$ & 75 & 14,31 & 3,77 & & & & \\
\hline & E. Civil & $\mathrm{S}$ & 88 & 13,91 & 4,06 & 0,31 & 142 & .758 & .00 \\
\hline & & $\mathrm{C}$ & 56 & 13,70 & 4,00 & & & & \\
\hline & Nivel & Graduado & 20 & 12,70 & 4,59 & 1,263 & 2 & .286 & .017 \\
\hline & Académico & Bachiller & 50 & 14,30 & 3,74 & & & & \\
\hline & & Universitario & 82 & 13,49 & 4,08 & & & & \\
\hline & Situación & Activo & 89 & 13,15 & 4,05 & $-0,996$ & 131 & .321 & .00 \\
\hline & Laboral & Parado & 44 & 13,89 & 3,99 & & & & \\
\hline
\end{tabular}




\begin{tabular}{|c|c|c|c|c|c|c|c|c|c|}
\hline \multirow[t]{11}{*}{ CAMI } & \multirow[t]{2}{*}{ Edad } & $<=35$ & 73 & 56,90 & 14,66 & \multirow{2}{*}{\multicolumn{2}{|c|}{$-1,411 \quad 143$}} & \multirow[t]{2}{*}{.160} & \multirow[t]{2}{*}{-.26} \\
\hline & & $>35$ & 72 & 60,50 & 16,01 & & & & \\
\hline & \multirow[t]{2}{*}{ Sexo } & $\mathrm{H}$ & 74 & 61,95 & 15,82 & \multirow[t]{2}{*}{2,787} & \multirow[t]{2}{*}{142} & \multirow[t]{2}{*}{.006} & \multirow[t]{2}{*}{.48} \\
\hline & & $\mathrm{M}$ & 70 & 54,97 & 14,11 & & & & \\
\hline & \multirow[t]{2}{*}{ E. Civil } & $\mathrm{S}$ & 83 & 56,55 & 15,02 & \multirow[t]{2}{*}{$-1,412$} & \multirow[t]{2}{*}{135} & \multirow[t]{2}{*}{.160} & \multirow[t]{2}{*}{-.27} \\
\hline & & $\mathrm{C}$ & 54 & 60,33 & 15,75 & & & & \\
\hline & \multirow{3}{*}{$\begin{array}{c}\text { Nivel } \\
\text { Académico }\end{array}$} & Graduado & 18 & 65,39 & 16,58 & \multirow[t]{3}{*}{2,074} & \multirow[t]{3}{*}{2} & \multirow[t]{3}{*}{.129} & \multirow[t]{3}{*}{.029} \\
\hline & & Bachiller & 47 & 57,81 & 13,41 & & & & \\
\hline & & Universitario & 79 & 57,44 & 15,95 & & & & \\
\hline & \multirow{2}{*}{$\begin{array}{c}\text { Situación } \\
\text { Laboral }\end{array}$} & Activo & 82 & 60,18 & 17,03 & \multirow[t]{2}{*}{0,768} & \multirow[t]{2}{*}{124} & \multirow[t]{2}{*}{.444} & \multirow[t]{2}{*}{.20} \\
\hline & & Parado & 44 & 57,91 & 13,28 & & & & \\
\hline
\end{tabular}

Los resultados arrojan diferencias de medias significativas en la escala MAKS que evalúa conocimientos en Salud Mental relacionado con estigma en las siguientes variables:

-Edad: Para el análisis estadístico se establece un punto de corte en los 35 años, encontrándose una diferencia significativa $p=.001$ entre los menores y los mayores de 35 , siendo los menores de 35 años los que cuentan con menos conocimientos en materia de Salud Mental relacionado con el Estigma. Además atendiendo al tamaño del efecto, se puede concluir que la edad es una variable que determina con un efecto moderado el conocimiento sobre el Estigma.

-Estado Civil: Existe una diferencia significativa $p=.002$ entre los solteros y los casados, siendo estos últimos los más informados sobre cuestiones de salud mental relacionadas con el Estigma, aunque el tamaño del efecto indica que la diferencia es pequeña.

En las Escalas CAMI (Actitudes) y RIBS (Conductas) encontramos diferencias significativas únicamente entre medias de los niveles de la variable Sexo. En ambas escalas tras aplicar una prueba $\mathrm{T}$ de Student para probar la diferencia de medias encontramos que tanto en el componente emocional del estigma (Actitudes-Prejuicios) como en la conductual (Intención de conducta estigmatizante-Conducta estigmatizante) son los hombres los que de forma significativa se diferencian presentando actitudes $p=.006$ e intención de conducta $p=.049$ más estigmatizantes que las mujeres. Aunque atendiendo al tamaño del efecto, se observa que en relación a las conductas el efecto es pequeño, y en relación a las actitudes, es moderado, más fuerte.

Un análisis correlacional entre MAKS, CAMI Y RIBS revela las siguientes correlaciones (Tabla 2): 
Tabla 2. Análisis Correlacional MAKS-RIBS-CAMI

\begin{tabular}{lccc}
\hline $\begin{array}{l}\text { Correlación } \\
\text { De Pearson }\end{array}$ & MAKS & RIBS & CAMI \\
\hline MAKS & 1 & $0,213^{* *}$ & $-0,006$ \\
\hline RIBS & $0,213^{* *}$ & 1 & $-0,543^{* *}$ \\
\hline CAMI &,- 006 & $-0,543^{* *}$ & 1 \\
\hline$*$ & La correlación es significativa al nivel 0,05 (bilateral). & \\
$* *$ & La correlación es significativa al nivel 0,01 (bilateral). &
\end{tabular}

Se puede observar una correlación negativa moderada entre las puntuaciones obtenidas en RIBS (conductas) y CAMI (actitudes) de $r=-.543 ; p=.001$, la cual expresa una relación donde a menos actitudes estigmatizantes, menos conductas estigmatizantes (al expresar mayores puntuaciones de CAMI mayores actitudes estigmatizantes y mayores puntuaciones de RIBS menores conductas estigmatizantes. La segunda correlación significativa, $r=213 ; p=.001$ aparece en las puntuaciones MAKS (conocimiento) y RIBS (conductas) sugiriendo que a más conocimientos sobre temas de salud mental, menos conductas estigmatizantes ( ya que mayores puntuaciones de MAKS apuntan a más conocimientos sobre salud mental libres de estigma y mayores puntuaciones de RIBS a menos conductas estigmatizantes).

\section{DISCUSIÓN}

Siguiendo la línea de investigación propuesta, necesitábamos comprender mejor el fenómeno del Estigma y sus distintos componentes de Conocimiento (Ignorancia), Actitudes (Prejuicio) y Conducta (Discriminación), en la población general.

Los resultados encontrados apuntan a diferencias significativas en la Escala de Conocimiento MAKS la cual mide factores de conocimiento sobre estigma en Salud Mental. Éstos nos indican que la población general de Almería presenta diferencias significativas en su conocimiento sobre materia de Salud Mental por grupos de edades, siendo los mayores de 35 años los mejor informados. Podría ser que las personas de más edad contaran con más número de experiencias que modificaran sus estereotipos y conocimientos estigmatizantes por otros que lo sean menos. O que hayan tenido acceso a fuentes de información más amplias y veraces. Así, estos resultados nos orientan en el diseño de futuras intervenciones que establezcan como población diana principalmente a los menores de 35 años, teniendo en cuenta los demás componentes de estigma y sus interacciones.

Por otro lado, se han encontrado diferencias significativas en actitudes y comportamiento en los diferentes grupos por sexo. Según los resultados obtenidos los hombres presentarían más actitudes e intención de conducta estigmatizantes en materia de salud mental que las mujeres de forma significativa, agu- 
dizándose esta diferencia en el caso de las actitudes-prejuicios ( $\mathrm{p}=.006)$. Estos resultados se podrán utilizar como guía en el diseño de intervenciones a aplicar a la población general.

Estas diferencias entre géneros podrían abrir una línea de investigación donde se ponga el foco en las mismas y se puedan examinar con más detalle, obteniendo conclusiones más específicas y concretas donde se tengan en cuenta elementos biológicos, antropológicos, sociales, culturales, conductuales o emocionales entre otros.

Según investigaciones anteriores, el Conocimiento es una variable mediadora y por sí misma determinante en las conductas de discriminación (Wolff et al., 1996). En nuestro estudio, nos proponíamos así mismo estudiar las relaciones entre los distintos componentes del Estigma en la población general. Los resultados indican que existe una relación significativa entre el Conocimiento y las Conductas, lo cual apunta a que mientras más conocimiento e información maneje la sociedad sobre salud mental, menos conductas estigmatizantes llevará a cabo aunque esta relación se retroalimenta y es circular por lo que habría en futuros estudios que determinar los mecanismos específicos en los que esta relación se manifiesta. Así mismo, hemos de destacar una moderada correlación entre Actitudes y Conducta según los resultados del test de correlación que sugiere que existe una relación entre ambos constructos en la dirección de que a menos actitudes (prejuicios) estigmatizantes, menos conductas estigmatizantes.

Según lo que nos indican los resultados y estudios anteriores, una intervención sobre la población masculina menor de 35 años de Almería capital que se enfocara a aumentar los conocimientos en factores determinantes para la Salud Mental en relación al estigma sería adecuada y podría influir en los otros dos componentes del Estigma.

Por otro lado, teniendo en cuenta que nos enfrentarnos a un fenómeno altamente complejo, la intervención debería ser multicomponente incluyendo elementos de intercambio de información y discusión así como interacción social, además del contenido puramente informativo. La implementación se plantea en trabajo conjunto bien con medios de comunicación, centros educativos u otros organismos con conocido impacto en la difusión de información y formación de la sociedad. La evaluación post intervención es un objetivo de futuros trabajos donde se seguirá estudiando este complejo fenómeno en sus diferentes componentes y la interacción entre estos para una mejor comprensión del estigma y sus mecanismos con la tarea de mejorar este campo de estudio y así ser capaces de diseñar intervenciones más específicas y efectivas.

\section{CONCLUSIONES}

1. Las personas de menor edad, menores de 35 años, poseen menos conocimientos sobre Salud Mental en relación al estigma.

2. Los hombres exhiben más actitudes (prejuicios) y conductas (intención) estigmatizantes que las mujeres. 
3. Existe una relación negativa entre conocimiento en materia de salud mental y conducta estigmatizante.

4. Existe una relación positiva entre actitudes estigmatizantes y conducta estigmatizante.

Se propone diseñar y aplicar intervenciones anti-estigma específicas y multicomponente haciendo énfasis, aunque no de forma exclusiva, sobre la población masculina menor de 35 años de Almería capital para aumentar sus conocimientos sobre salud mental en base a estos resultados, y modificar sus actitudes y conductas con respecto a los problemas de salud mental en relación al estigma. En siguientes estudios se llevará a cabo la evaluación de las intervenciones implementadas para poder evaluar el impacto de las mismas (tipo, intensidad, duración, componentes del Estigma) y seguir estudiando y comprendiendo el complejo fenómeno del Estigma.

\section{REFERENCIAS}

Brändli, H. (1999). The image of mental illness in Switzerland. En J. Guimon, W. Fischer, N. Sartorius (Eds.), The image of madness: the public facing mental illness and psychiatric treatment (pp. 29-37). Basel (CH): Karger.

Brea L. P., Pescolido, B. A., Martin, J. K., McLeod, J. D. y Jensen, P. D. (2007). Comparison of Public Attributions, Attitudes, and Stigma in Regard to Depression Among Children and Adults. Special Section on the National Stigma Study-Children. Psychiatric Services, 58, 632-635.

Corrigan, P. W. y Watson, A. C. (2002). The impact of stigma on people with mental illness. World Psychiatry, 1, 16-19.

Corrigan, P. W., Edwards, A. B., Green, A., Diwan, S. L. y Penn, D. L. (2001). Prejudice, Social distance and Familiarity with Mental Illness. Schizophrenia Bulletin, 27, 219-225.

Evans-Lacko, S., Rose, D., Little, K., Rhydderch, D., Henderson, C. y Thornicroft G. (2011). Development and Psychometric Properties of the Reported and Intended Behaviour Scale (RIBS) Epidemiology and Psychiatric Sciences, 20, 261-271.

Evans-Lacko, S., Little K., Meltzer H., Rose D., Rhydderch D., Henderson C. y Thornicroft G. (2010). Development and Psychometric Properties of the Mental Health Knowledge Schedule (MAKS). Canadian Journal of Psychiatry, 55, 440-448.

Evans-Lacko, S., London, J., Little, K., Henderson, C. y Thornicroft, G. (2010). Evaluation of a brief anti-stigma campaign in Cambridge: do short-term campaigns work? BMC Public Health, 10, 339.

Fazio, R. H., Olson, M. A. (2003) Attitudes: Foundations, Functions, and Consequences. En M. A. Hogg, J. Cooper (Eds.). The Sage Handbook of Social Psychology (pp. 139-160). Londre: Sage.

López, M. (2012). El Estigma en Salud Mental. Psychology, Society, \& Educa- 
tion, 4, 131-136.

López, M., Laviana, M., Fernández, L., López, A. Rodríguez, A.M. y Aparicio, A. (2008). La Lucha contra el Estigma y la Discriminación en Salud Mental. Una estrategia compleja basada en la información disponible. Revista de la Asociación Española de Neuropsiquiatría, 28, 43-83.

Magliano, L., Read, J., Patalano, M., Sagliocchi, Oliviero, N., D’Ambrosio, A. y Cerrato, F. (2012) Contrarrestar el estigma hacia las personas con esquizofrenia en el ámbito sanitario: una experiencia piloto en una muestra de estudiantes italianos de medicina. Psychology, Society, \& Education, 4, 169-181.

Muñoz, M., Pérez Santos, E., María Crespo, M. y Guillén, A.I. (2009). Estigma y Enfermedad Mental: Análisis del rechazo social que sufren las personas con enfermedad mental. Madrid: Editorial Complutense.

Taylor, S. M., y Dear, M. J. (1981). Scaling community attitudes toward the mentally ill. Schizophrenia Bulletin, 7, 225-240.

Thorndicroft, G. (2006). Discrimination against people with mental illness. Oxford: Oxford University Press.

Wolff G., Pathare S. y Craig T. (1996). Community Knowledge of mental illness and reaction to mentally ill people. British Journal of. Psychiatry, 168, 191198. 